\title{
Evaluation of Mathematics Teacher Candidates' the Ellipse Knowledge According to the Revised Bloom's Taxonomy
}

\author{
Aytaç Kurtuluş ${ }^{1, *}$, Tuba Ada ${ }^{2}$ \\ ${ }^{1}$ Faculty of Education, Eskişehir Osmangazi University, Turkey \\ ${ }^{2}$ Faculty of Education, Anadolu University, Turkey
}

Copyright $\mathrm{O} 2017$ by authors, all rights reserved. Authors agree that this article remains permanently open access under the terms of the Creative Commons Attribution License 4.0 International License

\begin{abstract}
In this study, the teacher candidates who learnt to find the algebraic equation corresponding to geometric structure of the ellipse in analytic geometry classes were requested to find the algebraic representations corresponding to the structures that contained ellipses in different positions. Thus, it would be possible to determine higher order thinking skills of the teacher candidates' ellipse knowledge in the applying, analyzing, evaluating and creating categories according to the Revised Bloom's Taxonomy. The results showed that the teacher candidates in the Applying category completed the Remembering and Understanding categories since the learning outcomes in cognitive learning domain regarding the ellipse knowledge are in hierarchical order. Therefore, the teacher candidates' overall achievement level was in the Applying category at minimum. In addition, the results revealed that about two-thirds of the teacher candidates were in Analyzing, Evaluating or Creating categories of higher-order cognitive learning domain in terms of analytically examining ellipses.
\end{abstract}

Keywords Analytical Examination of the Ellipse, Higher Order Thinking Skills, Mathematics Pre-service Teacher

\section{Introduction}

A locus is a set of points that satisfy certain conditions. These points form a geometric structure of a figure. Equations are used to determine the properties of geometric structures. To do this, each point in the plane is coordinated using Cartesian coordinates and the coordinates of points that define geometric structures are determined. Geometric figures can be characterized by algebraic equations in this way. The locus of the ellipse is defined as a set of points whose sum of distances to two fixed points is constant [7].
In this study, the teacher candidates who learnt to find the algebraic equation corresponding to geometric structure of the ellipse in analytic geometry classes were requested to find the algebraic representations corresponding to the structures that contained ellipses in different positions. Thus, it would be possible to determine higher order thinking skills of the teacher candidates' ellipse knowledge in the Applying, Analyzing, and Evaluating and Creating levels according to the Revised Bloom's Taxonomy.

As in every course, it is important to determine the extent to which learning outcomes are achieved in cognitive processes in mathematics classes. In the taxonomy of cognitive learning domains that he developed in 1956, Bloom identified the level of cognitive learning in one dimension as Knowledge, Comprehension, Application, Analysis, Synthesis and Evaluation [10]. Then this taxonomy was revised in line with the new approaches in education [2]. In the revised version of the taxonomy, cognitive learning domain was considered to consist of two dimensions: knowledge and cognitive process. In the revised version, knowledge indicated the content of objectives (outcomes) and consisted of four types of knowledge: Factual, Conceptual, Procedural and Metacognitive. In cognitive process, on the other hand, the names in the six categories were changed from noun to verb forms. Also, in this dimension, because the synthesis level included evaluation in the synthesis and evaluation, the last two levels in the original form of the taxonomy, and there was no consensus about the hierarchy, the order of these two categories was reversed [1,11]. Therefore, the six categories named as Knowledge, Comprehension, Application, Analysis, Synthesis and Evaluation in the original taxonomy were respectively named in verb forms as Remembering, Understanding, Applying, Analyzing, Evaluating and Creating [3, 4,9,11]. Of these categories, the first three Remembering, Understanding and Applying - were described as lower-order skills while the other three - 
Analyzing, Evaluating and Creating - were described as higher-order thinking skills. The revised form of the taxonomy is expected to contribute to development and measurement of skills such as mathematical modeling and problem solving and to serve as an important assessment tool in terms of revealing higher-order thinking skills that reflect a constructivist teaching approach [4].

In the light of these, the aim of this study was to explore how mathematics teacher candidates employed their knowledge on the ellipse in the Applying category, a lower order category in the cognitive process dimension, and in the Analyzing, Evaluating and Creating categories, higher-order categories in the cognitive process dimension.

\section{Methods}

\subsection{Research Model}

The qualitative study used the descriptive analysis method. In this approach, the data are classified, summarized and interpreted according to pre-determined themes [8].

\subsection{Study Sample}

This study was conducted with a total of 106 third year teacher candidates studying Primary School Mathematics Teaching of Faculties of Education at a state university in Turkey during the fall semester of 2015-2016 academic years. They took the Analytic Geometry course which consist of conic sections subjects(ellipse, hyperbola, parabola) given by the researcher.

\subsection{Data Collection Tool}

A test about "Analytical Examination of the Ellipse" was used in order to determine how the teacher candidates used their knowledge on the ellipse in the Applying, Analyzing, Evaluating and Creating categories.

\subsection{Data Analysis}

Solutions made by the participants were analyzed by the researchers based on the basic principles established according to the cognitive process categories of the taxonomy. The researchers individually made descriptive analyzes of the written documents of the teacher candidates' written answers. A total of 106 solutions where there was agreement between the researchers were analyzed.

The inter-rater reliability of Analytical Examination of the Ellipse Test was found by testing the tool on 106 teachers candidates in the study. The teacher candidates were given code numbers and their levels of cognitive process were determined based on the analysis results. The researchers' results were compared. The inter-rater reliability of the research was calculated using the following formula [5]; reliability=number of agreements/(total number of agreements + disagreements)

The research reliability was found to be 0,93 .

\subsection{Implementation Process}

This stage of the study focused on learning outcomes on the ellipse in the topic of conic sections in Analytical Geometry course:

Examine and practice the rules of the ellipse analytically,

- Define the major and minor axes of the ellipse; calculate the lengths of these axes,

- Estimate certain points of the ellipse such as the coordinates of the vertices and focal points of the ellipse, the distance between the focuses, and the eccentricity of the ellipse,

- Formulate the equation for an ellipse centered or not centered at the origin and define all the properties of that ellipse.

In accordance with these learning outcomes, the teacher candidates were thoroughly informed about the analytical examination of the ellipse and basic practice questions. As a part of the course, all ellipses that were centered or not centered at the origin and had $\mathrm{x}$-axis or $\mathrm{y}$-axis as major axis were presented with examples through algebraic equations and graphs with geometric representations.

During this stage, the teacher candidates were found to be successful in the Applying level of the lower-order skills of the cognitive process. In order to determine how the participants made use of their knowledge of the ellipse in the Analyzing, Evaluating and Creating categories, or higher-order skills in other words, the teacher candidates were asked to reflect on and solve the following practice questions in the measurement-evaluation stage.

The participants were first asked to plot the multiple geometric structures given in Figure 1 on a coordinate system.

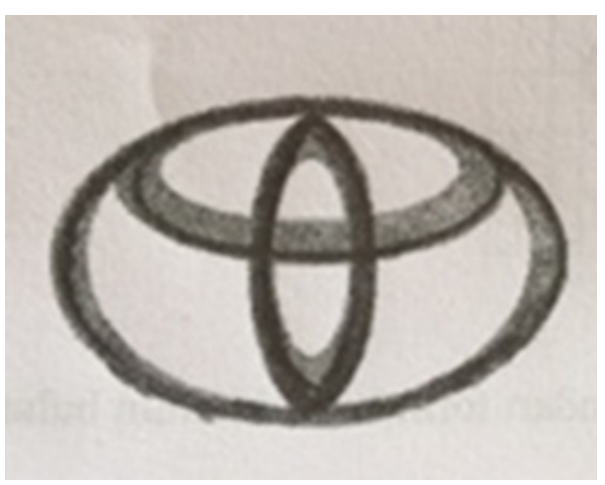

Figure. 1. The image of multiple geometric structure in Question 1 and 2

In Question 1, they were asked to write the equations specifying the data required for writing the equation of each ellipse in the figure 1. In Question 2, they were asked to find the eccentricity of each of the ellipses and compare 
their flatness.

In this study, according to the Revised Bloom's Taxonomy, cognitive learning domain was considered to cognitive process dimension. The cognitive process dimension shows how learning outcomes are realized.

The learning outcomes in the cognitive process are realized in six categories:

- Remembering: Recalling the definition of the ellipse and the vertices, focuses and eccentricity required for estimating its equation.

- Understanding: Interpreting whether an ellipse is centered on the origin or not by giving examples of the equation and graph of the ellipse, and finding the components forming the eccentricity.

- Applying: For a given problem situation, finding the equation for the ellipse using the previously learnt method, sketching the graph for the ellipse in accordance with the method by considering the main components of the ellipse, and finding the eccentricity by using the required data for multiple interrelated ellipses.

- Analyzing: For a graph situation including multiple ellipses as a whole, finding the equations for these ellipses by determining the major/minor axis and focus of each through establishing a relationship between the whole and the ellipses making up the whole, and comparing their eccentricities.
- Evaluating: Reaching a conclusion by critiquing the relative positions of the ellipses forming the whole, and comparing and contrasting their eccentricities and interpreting their flatness.

- Creating: Developing a solution by choosing an original coordinate system and justifying abstract relationships and ellipse equations by using letters in the original coordinate system and the solution in terms of eccentricities.

Since the aim of this study was to explore how mathematics teacher candidates employed their knowledge of the ellipse in the Applying, Analyzing, Evaluating and Creating categories.

\section{Results}

The learning activities and actions of Practice Question 1, where the participants were asked to formulate the equations by specifying the data required for writing the equation of each ellipse in Figure 3, according to the Revised Bloom's Taxonomy are given in Table 1. How the teacher candidates used their knowledge of the ellipse based on the categories in the cognitive process for Question 1 was analyzed descriptively and the distribution of the participants according to these categories is shown in Table 1.

Table 1. The descriptive analysis table according to the revised Bloom Taxonomy

\begin{tabular}{|c|c|c|c|}
\hline & Learning activities & Teacher Candidates & Frequency \\
\hline Creating & $\begin{array}{l}\text { Choose different original coordinate systems that represents } \\
\text { the geometric situation and relationship, develop the } \\
\text { equations through assigning letters on the coordinate system, } \\
\text { find and interpret the eccentricity }\end{array}$ & $\begin{array}{c}\mathrm{A} 14, \mathrm{~A} 16, \mathrm{~A} 22, \mathrm{~A} 40, \mathrm{~T} 37, \mathrm{~T} 38, \mathrm{~T} 18, \mathrm{~T} 27, \mathrm{~T} 19, \\
\mathrm{~T} 23\end{array}$ & 10 \\
\hline Evaluating & $\begin{array}{l}\text { Reaching a conclusion by critiquing the relative positions of } \\
\text { the ellipses forming whole in the standard coordinate } \\
\text { system, and comparing and contrasting their eccentricities }\end{array}$ & $\begin{array}{l}\text { A3, A6, A13, A15, A17, A20, A24, A27, A32, A33, } \\
\text { A34, A35, A43, T15, T19, T27, T28, T29, T31, } \\
\text { T33, T34, T35, T36, T37, T38, T39, T40, T41, T42, } \\
\text { T44, T45, T46, T47, T50, T54, T55, T58, T60, T62, } \\
\text { T64, T 67, T68, T69, T71, T74, T78, T80 }\end{array}$ & 47 \\
\hline Analysing & $\begin{array}{l}\text { For a given geometric situation, correctly find the equations } \\
\text { for three ellipses by considering the relative relationships of } \\
\text { only two ellipses, or correctly find the equations for three } \\
\text { ellipses without considering their relative relationships of } \\
\text { only two ellipses, interpret the eccentricity }\end{array}$ & $\begin{array}{c}\text { A1,A2, A4, A8, A9, A10, A12, A19, A25, A26, } \\
\text { A36, A37, A41, A42, A47, A23,T14, T17, T30, } \\
\text { T32, T49, T51, T53, T56, T63, T66, T72, T73, T76, } \\
\text { T77,T81 }\end{array}$ & 31 \\
\hline Applying & $\begin{array}{l}\text { Correctly find the equation for only an ellipse centered at the } \\
\text { origin by plotting it on the coordinate plane, find the } \\
\text { eccentricity }\end{array}$ & $\begin{array}{l}\text { A5, A7, A11, A28, A30, A31, A38, A39, A44, } \\
\text { A45, A46,A48, T48,T52, T59, T65, T75, T79 }\end{array}$ & 18 \\
\hline Understanding & $\begin{array}{l}\text { Find the equation when given a single ellipse, find the } \\
\text { eccentricity of an ellipse by determining the major axis and } \\
\text { the focus specified in the formula }\end{array}$ & & - \\
\hline Remembering & $\begin{array}{l}\text { Define the ellipse, determine the vertices and focuses } \\
\text { required for finding the equation, find the eccentricity for an } \\
\text { ellipse when given the major axis and the focus }\end{array}$ & & - \\
\hline
\end{tabular}




\subsection{Results Obtained in the Creating Category}

The descriptive analysis identified 10 participants in the Creating category of higher-order cognitive learning domain (Table 1). These teacher candidates were evaluated to be in this category since they reached a solution by using an original coordinate system and found abstract relationships and ellipse equations by using letters in the coordinate system. The solutions made by four of the teacher candidates in this category were directly quoted and interpreted below. Equation as normal text:

Sample Solution by Teacher Candidate TC38 in the Creating Category

Teacher Candidate TC38 planned and plotted the centers of the ellipses at any point in the coordinate plane instead of the origin (Figure 2). 1. The participant plotted the centers of the ellipses that s/he called Ellipse 1 and Ellipse 3 on the same major axis but plotted the center of Ellipse 2 on the major axis of Ellipse 3. S/he determined the coordinates of the vertices on the shape for each of the ellipses. For Ellipse 1, $\mathrm{s} / \mathrm{he}$ found the values $\mathrm{a}=5$ and $\mathrm{b}=4$ using the major axis length (2a) and the minor axis length (2b). S/he then found $c=3$. Therefore, the participant formulated the equation for Ellipse 1 by determining the vertices required for writing it.

The participant assumed that Ellipse 2 was at $(8,12)$ and $(8,8)$ points and centered at $(8,10)$. Therefore, $s /$ he formulated the equation for Ellipse 2 by assuming $a=3, b=2$.

$\mathrm{S} /$ he formulated the equation for Ellipse 3 by assuming its center the same as that of Ellipse 1 and finding the values $a=4$ and $b=2$. As can be seen in the question in Figure 5, the centers for these two ellipses were the same, but their focuses were on the different axes. Also, in the equation formulated by the participant, the major axis of Ellipse 3 was in parallel to the y-axis.

Using this strategy, the participant correctly formulated the algebraic equations for the ellipses by coordinating their geometric shapes.

\section{Sample Solution by Teacher Candidate A22 in the Creating Category}

Teacher Candidate A22 plotted the ellipses on the coordinate system as shown in Figure 3. The participant stated that it was necessary to know the vertices of the ellipse to formulate its equation. However, instead of assigning special values to them in the coordinate system, s/he assigned letters and found the centers, vertices and focuses of the ellipses in this way.

For the letters s/he used when finding the equation for the ellipse in bold in Figure 3, the participant highlighted the relationship $\mathrm{a}>\mathrm{b}$ between the major axis (2a) and minor axis (2b) lengths. In addition, s/he formulated the equation by stating that the major axis of that ellipse was $\mathrm{x}$-axis. S/he found the distance between the focuses required for finding the eccentricity. Using the ratio of distance between the focuses to the major axis length, s/he obtained the eccentricity.

When finding the equation for the ellipse in plain lines, the participant assumed the minor axis length of the ellipse in bold to be equal to the major axis length of the ellipse in plain lines. In this way, $s /$ he obtained the relationship $b>c$ by taking the major axis length as $2 \mathrm{~b}$ and the minor axis length as 2c (Figure 3). S/he then found the distance between the Using the ratio of distance between the focuses to the major axis length, s/he obtained the eccentricity.

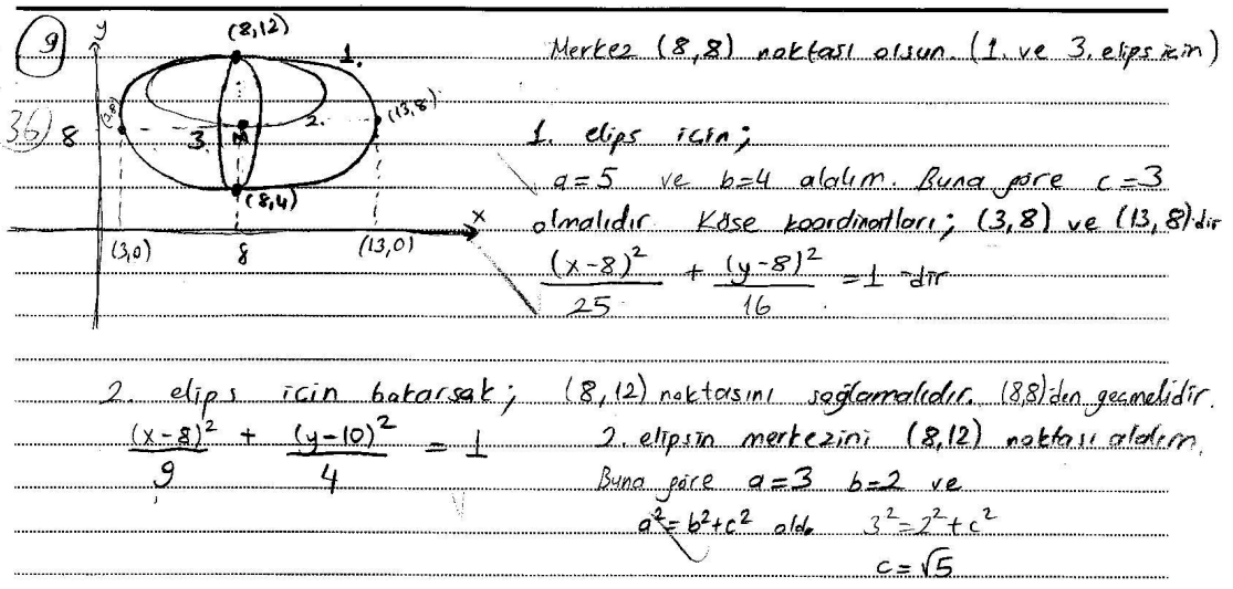

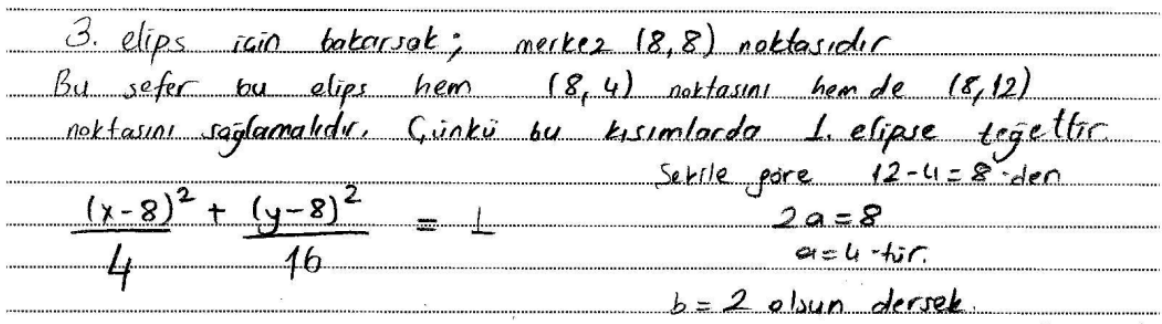

Figure 2. Sample Solution by Teacher Candidate TC38 


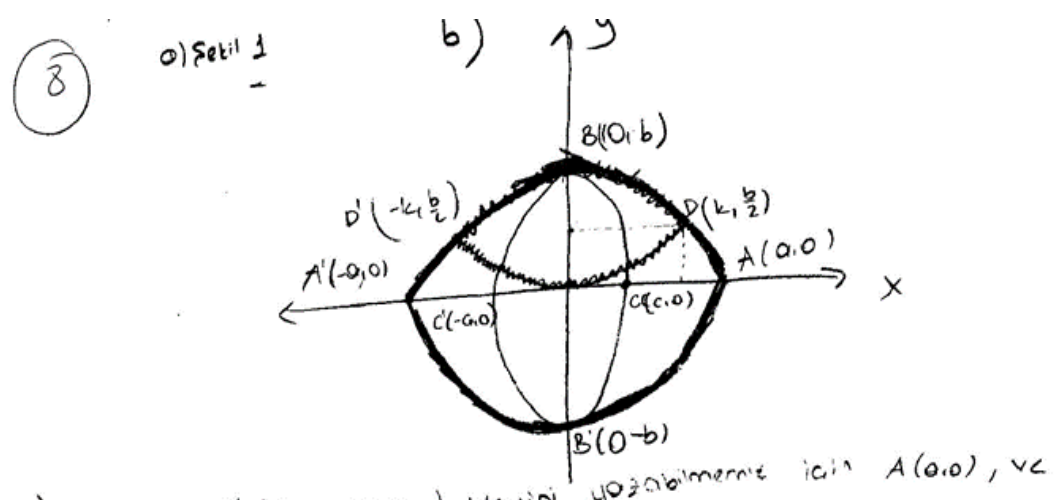

c) royu ile cizilnis. elips duklewini yoz

$B(0, b)$ noktanina intigocimiz vordr. $\frac{x^{2}}{a^{2}}+\frac{y^{2}}{b^{2}}=1$ olocik yozur ve $\quad$ oldigu icin $|a|>|b|$ Aak rerki elips iain geekli olen noktaler. $B(0, b), C(c, 0)$ $|b| s|c|$ ve elips derkleni $\frac{y^{2}}{b^{2}}+\frac{x^{2}}{c^{2}}=1$ 'dr $e_{1}=\frac{\sqrt{a^{2}-b^{2}}}{a} \quad|a|>|b|$
$e_{2}=\frac{\sqrt{b^{2}-c^{2}}}{b} \quad \therefore|b|>|c|$
$e_{3}=\frac{\sqrt{a k^{2}-b^{2}}}{2 k} \quad|k|>\left|\frac{b}{2}\right| \Rightarrow|2 k|>|b|$ Burbasikiticia kosilastrabilmensit tian ya nokter bilmemit

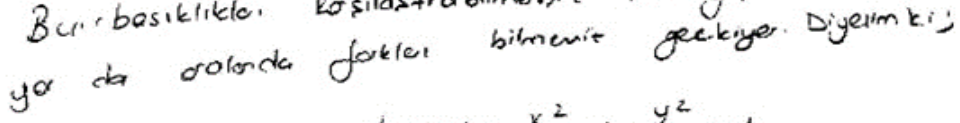
Koyu cizilmis ellps denkteni; $\frac{x^{2}}{64}+\frac{y^{2}}{36}=1$

$$
\begin{aligned}
& a=8 \\
& b=6 \quad \text { ofr. } \\
& 64=36+d^{2}=\sqrt{28}
\end{aligned}
$$

Aark reikt: elips ian; $\frac{y^{2}}{30}+\frac{x^{2}}{9}=1$

$$
\begin{aligned}
36 & =9-d^{2} \\
36 & c_{2}=\frac{\sqrt{27}}{6} \\
& =27 . \sqrt{77}
\end{aligned}
$$$$
\text { Trtike. elips ien } \frac{x^{2}}{16}+\frac{(y-3)^{2}}{9}=1 \quad \text { es }=\frac{\sqrt{7}}{4}
$$

$$
\begin{gathered}
16^{\prime}=9-\theta^{2} \\
\theta=\sqrt{7}
\end{gathered}
$$$$
\ell_{1}=c_{3}>e_{2} \text { oler Ama jutpordaki degorer iar. }
$$

Figure 3. Sample Solution by Teacher Candidate A22 
When finding the equation for the ellipse in dashed lines in Figure 3, the participant took the minor axis length as the half of the minor axis length of the ellipse in bold (2b). Therefore, $\mathrm{s} /$ he formulated the equation for the ellipse by taking the major axis length of this ellipse as $2 \mathrm{k}$ and the minor axis length $a$ s $b$ and taking its center at $(0, b / 2)$. After that, the participant found the distance between the focuses. Using the ratio of distance between the focuses to the major axis length, $\mathrm{s} /$ he found the eccentricity.

In this way, the participant found the eccentricities of each of the ellipses. Stating that it was necessary to know the points to compare and contrast the eccentricities, s/he assigned special values to the vertices of the ellipses. For these special values, $\mathrm{s} / \mathrm{he}$ found $e_{1}=e_{3}>e_{2}$ and stated that the flatness of Ellipses 1 and 3 was equal and flatter than that of Ellipse 2.

Instead of assigning special values in the coordinate system, Teacher Candidate A22 used letters. The participant used letters by determining the relationships between the major and minor axes of the ellipses. Also, s/he correctly formulated the equations for each of the ellipses by taking these relationships into consideration. Finally, this participant was evaluated to be in the Creating category since $\mathrm{s} /$ he interpreted the results by comparing and contrasting the eccentricities of the ellipses.

\subsection{Results Obtained in the Evaluating Category}

The descriptive analysis identified 47 participants in the Evaluating category of higher-order cognitive learning domain (Table 1). These teacher candidates were evaluated to be in this category since they reached a conclusion by critiquing the relative positions of the ellipses forming the whole and interpreted the eccentricities of the ellipses by comparing and justifying them. The solutions made by two of the teacher candidates in this category were directly quoted and interpreted below.

\section{Sample Solution by Teacher Candidate A24 in the Evaluating Category}

Teacher Candidate A24 plotted the shape in a coordinate system as shown in Figure 4. When determining the points where the ellipses intersected the axes, the participant made choices at certain ratios by taking the relative positions of the ellipses into consideration (Figure 4).
9)

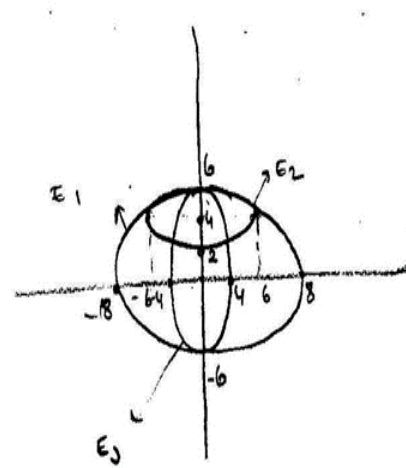

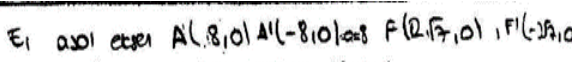

yede exxey $B\left(0,618^{\prime}(0,-6)=b=b\right.$

$a^{2}=b^{2}+c^{2} \quad 64=3 b+c^{2}$

$c^{2}=28 \quad c=\mp 25$

$\frac{x^{2}}{0^{2}}+\frac{y^{2}}{b^{2}}=1+0$ etern $x$

$E_{1} \rightarrow \frac{x^{2}}{64}+\frac{y^{2}}{36}=1$

$E_{2} \operatorname{mokez}(0,4)$

$E_{2}$ aral atsen $A_{2}(-6,4)$
$E_{2}$ yeder exing $(0,6)$

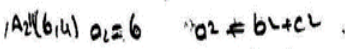

$18(0,2) \quad b=2 \quad 36=4+c 6$.

$E_{2}+\frac{x^{2}}{26}+\frac{(y-4)^{2}}{44}=1$

Es moker $(0,0)$

E) osp ersen Ab $(0,6)$

E) yedet ersa $B_{3}(4,0)$

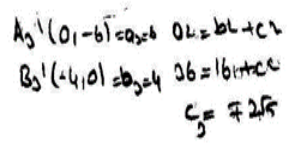

$\varepsilon_{3}+$

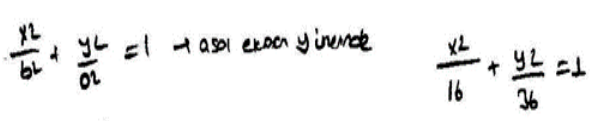

d) mukelite $=\frac{c}{a}=e$

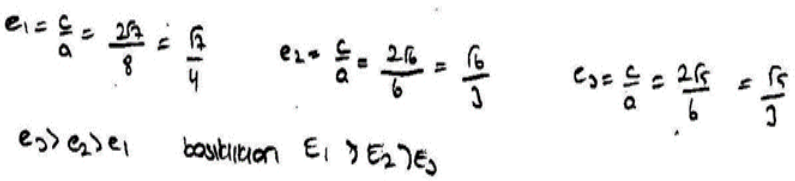

Figure 4. Sample Solution by Teacher Candidate A24 
$\mathrm{S} /$ he found the lengths of major and minor axes by determining the coordinates of the vertices of the larger ellipse $\left(E_{1}\right)$ first. In this way, $\mathrm{s} /$ he found the equation for the $E_{1}$ ellipse centered at the origin. The participant secondly determined the center and the lengths of major and minor axes of the smaller horizontal ellipse $\left(E_{2}\right)$, according to $E_{1}$ ellipse. Finally, by stating that the major axis of the vertical ellipse $\left(E_{3}\right)$ was y-axis and the major axis length of this ellipse was equal to the minor axis length of $E_{1}$ ellipse.

The participant found the distance between the focuses, which was required for finding the eccentricities of the ellipse by using the lengths of the major and minor axes that $\mathrm{s} /$ he determined for each of the ellipses. The participant then interpreted the eccentricity of each of the ellipses by estimating each eccentricity based on the ratio of the distance between the focuses to the major axis length.

When the compatibility of the equations formulated by Teacher Candidate A24 with the given geometric situation was checked with Geogebra, the graph in Figure 5 was obtained.

Teacher Candidate A24 was evaluated to be in the Evaluating category since s/he reached a judgment based on the relationships between the ellipses and interpreted the eccentricities.

\section{Sample Solution by Teacher Candidate A20 in the Evaluating} Category

Figure 6 shows the answer given to this question by Teacher Candidate A20. This participant plotted the ellipses on a standard coordinate system. S/he first determined proportional values for $\mathrm{a}, \mathrm{b}$ and then formulated the equations for the ellipses by taking the centers of each of the ellipses. By finding $\mathrm{c}, \mathrm{s} / \mathrm{he}$ found the eccentricity of each of the ellipses. After comparing and contrasting the results, $\mathrm{s} / \mathrm{he}$ found the relationship $e_{\theta}>e_{\beta}>e_{\alpha}$ among the eccentricities of the ellipses s/he called $\theta, \beta, \alpha$ respectively.

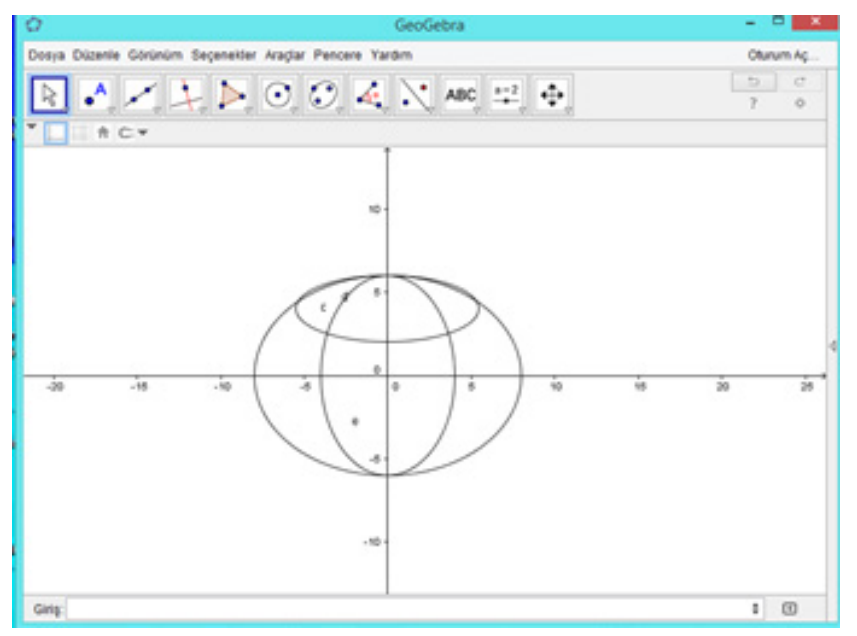

Figure 5. Ggraph of geometric structure formed by Teacher Candidate A24 with the Geogebra software

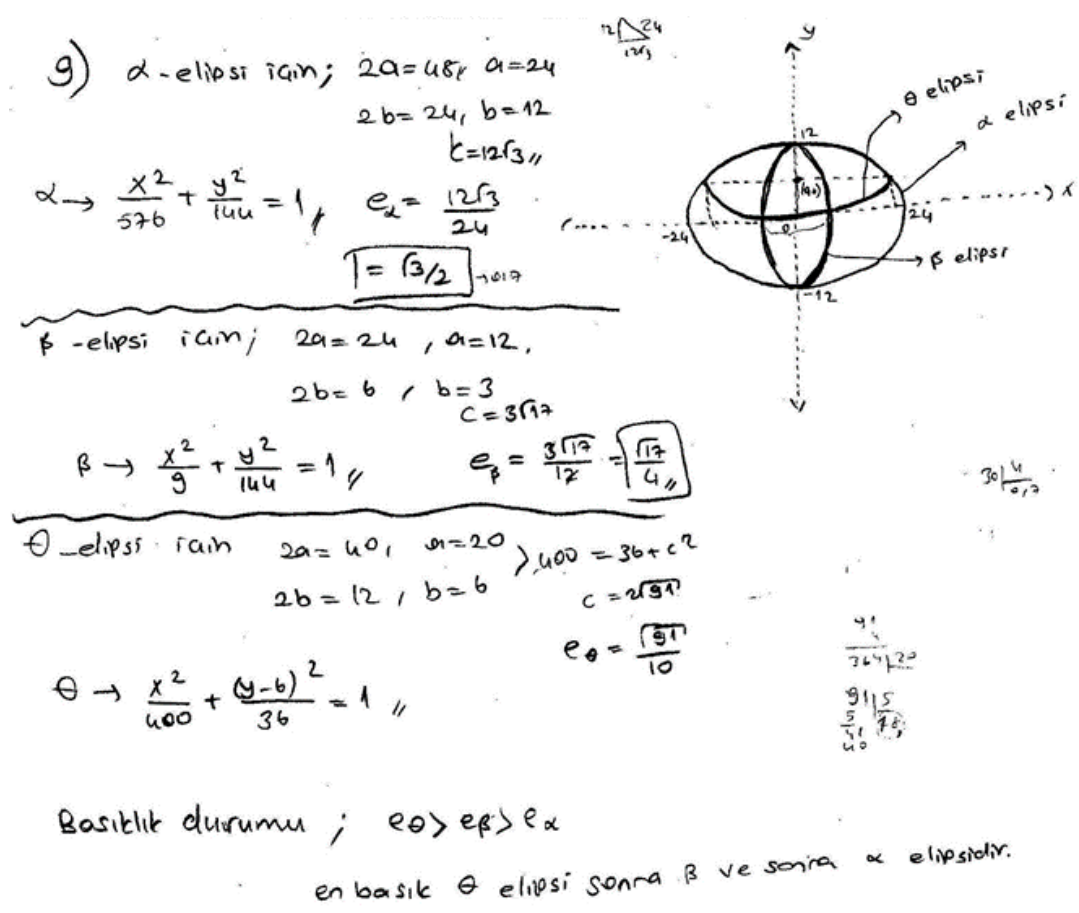

Figure 6. Sample Solution by Teacher Candidate A20 
The participants in this category firstly assigned proportional values to the vertices in a coordinate system that they chose by taking their relative positions into account as well. Next, by considering the location of the major axis of the ellipses being on the $\mathrm{x}$-axis or $\mathrm{y}$-axis, they first found the equations for the ellipses that were centered at the origin and then found the equation for an ellipse that was not centered at the origin so that it would be compatible with the others. Since the students were aware that the lengths of major and minor axes were important for an ellipse, they took care to proportionally select them on the coordinate system first. Also, since they were aware that it was necessary to know the distance between the focuses to determine the eccentricity, they performed the necessary calculations by using the lengths of the major and minor axes. Finally, they were able to interpret eccentricity as the flatness of the ellipse.

\subsection{Results Obtained in the Analyzing Category}

A total of 31 teacher candidates in the Analyzing category of higher-order cognitive domain compared and contrasted the eccentricities of the ellipses by finding the equations, major and minor axes, vertices and focus of each of the ellipses without considering the relationship between the whole and the parts of the whole in a graph situation containing multiple ellipses. They were also evaluated to be in this category since they compared the eccentricities of the two ellipses by correctly finding their equations based on their relative positions. The solutions made by two of the teacher candidates in this category were directly quoted and interpreted below.

\section{Sample Solution by Teacher Candidate A10 in the Analyzing Category}

Teacher Candidate A10 used a standard coordinate system and plotted it on the center of the larger ellipse outside (Figure 7). Taking the relative positions of the ellipses in the coordinate system into consideration, the participant then found the points where the axes were intersected. After that, $\mathrm{s} / \mathrm{he}$ found the coordinates of the focuses of the major and minor vertices of the ellipses and their centers based on the lengths of the major and minor axes for each of the ellipses. $\mathrm{S} /$ he also correctly found the equations for the ellipses by considering that the horizontal ellipse and the vertical ellipse were centered at the origin and the major axis of the horizontal ellipse was on the $y$-axis. On the other hand, s/he determined the center of the third horizontal ellipse as $(0,2)$. Therefore, the teacher candidate failed to reach the Evaluating category and $\mathrm{s} / \mathrm{he}$ was evaluated to be in the Analyzing category since s/he did not check the relationship between the results as a whole that $\mathrm{s} / \mathrm{he}$ obtained by breaking it into smaller parts.

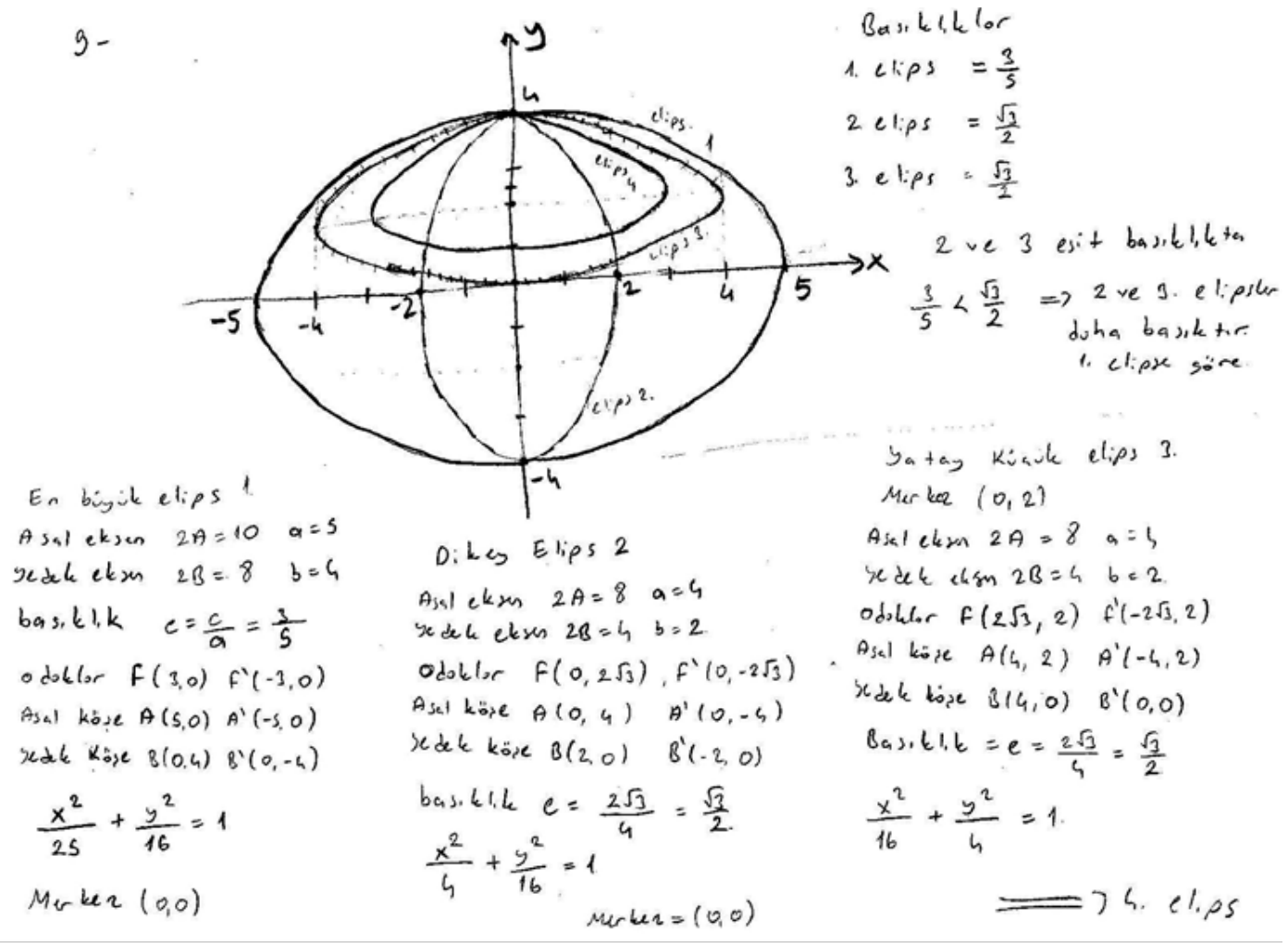

Figure 7. Sample Solution by Teacher Candidate A10 
Teacher Candidate A10 took the relationship between the equations of the two ellipses into account, but $\mathrm{s} /$ he failed to consider the relationship for the third equation that $\mathrm{s} / \mathrm{he}$ found. Figure 8 shows a screenshot of the relative positions of the equations found by the participant.

\section{Sample Solution by Teacher Candidate A23 in the Analyzing Category}

Teacher Candidate A23 started the solution by plotting a coordinate system on the given geometric shape, but s/he broke the whole into smaller parts and performed plotting on a separate coordinate system for each of the ellipses. The participant then correctly found the equations by determining the vertices for each of the ellipses (Figure 9).

(9)

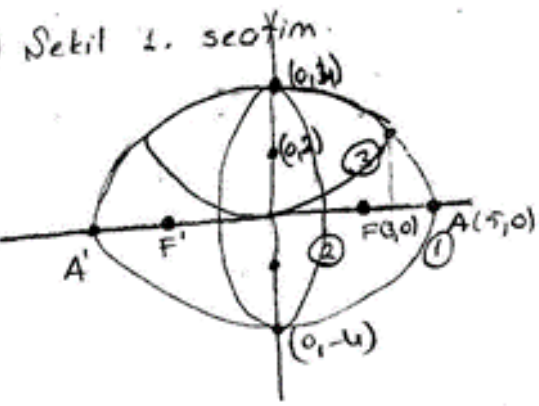

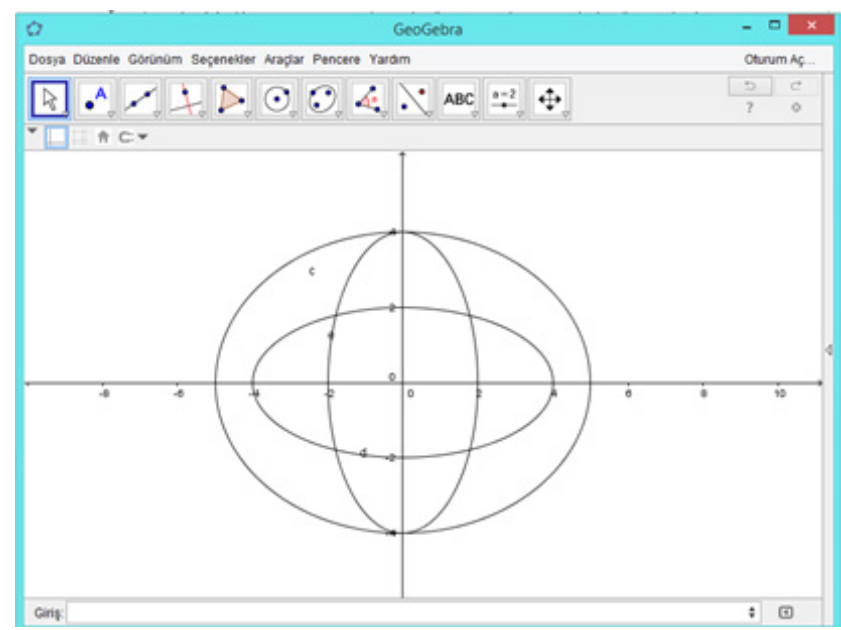

Figure 8. Ggraph of geometric structure formed by Teacher Candidate A10 with the Geogebra software

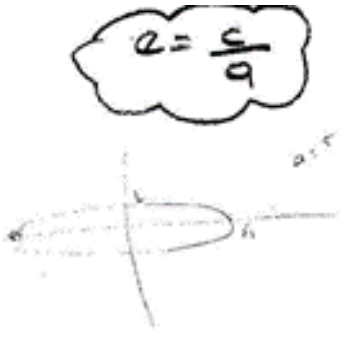

$\ngtr$

(1). elips iain bilinmesi gerekenter

$\frac{x^{2}}{a^{2}}+\frac{y^{2}}{b^{2}}=1$

$a^{2}=b^{2}+c^{2}$

$25=b^{2}+9$

$b=4 \quad a=5 \quad c=3$

(1). elipsin dis merkglipi

$F^{\prime}(-3,0)$

$\begin{array}{ll}A(5,0) & B(0,4) \\ A^{\prime}(-5,0) & B^{\prime}(0,-4)\end{array}$

(1) $\frac{x^{2}}{25}+\frac{y^{2}}{16}=1$

$2 a=10 \rightarrow$ asal eksen yuslupi 4

$b$ intset

$2 b=4 \rightarrow$ yedet

eksen uzunlupu

te buyuidikue basiklik ortor:

$$
\hat{T} e_{i}=\frac{c}{\sqrt{0}}=\frac{3}{5}=\frac{9}{15} d i r
$$

\section{(2). alrps icin bilinmesi gerekenier.}
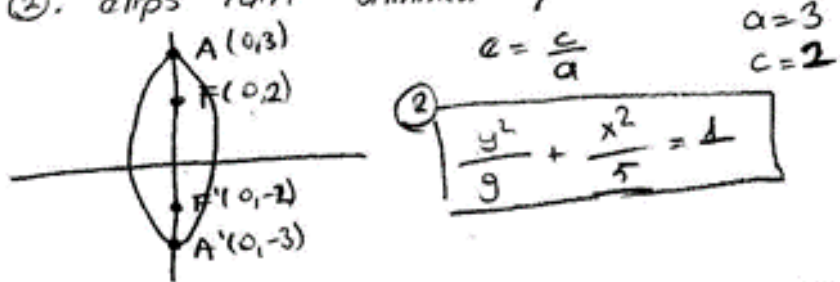

$$
\begin{aligned}
a^{2} & =b^{2}+c^{2} \\
9 & =b^{2}+4 \\
b & =\sqrt{5} \\
2 a=6 & \rightarrow \text { asal eksen } \\
2 b=2 \sqrt{5} & \rightarrow \text { yedek cksen }
\end{aligned}
$$

$$
c_{2}=\frac{c}{a}=\frac{2}{3}-\frac{10}{15}
$$

$a_{2}>e_{1} \Rightarrow$ Dolayisiyla (2) elips (1).

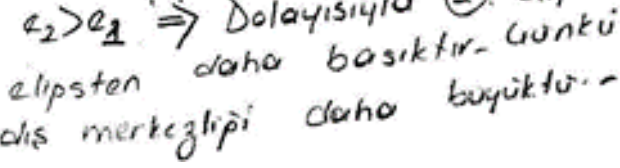




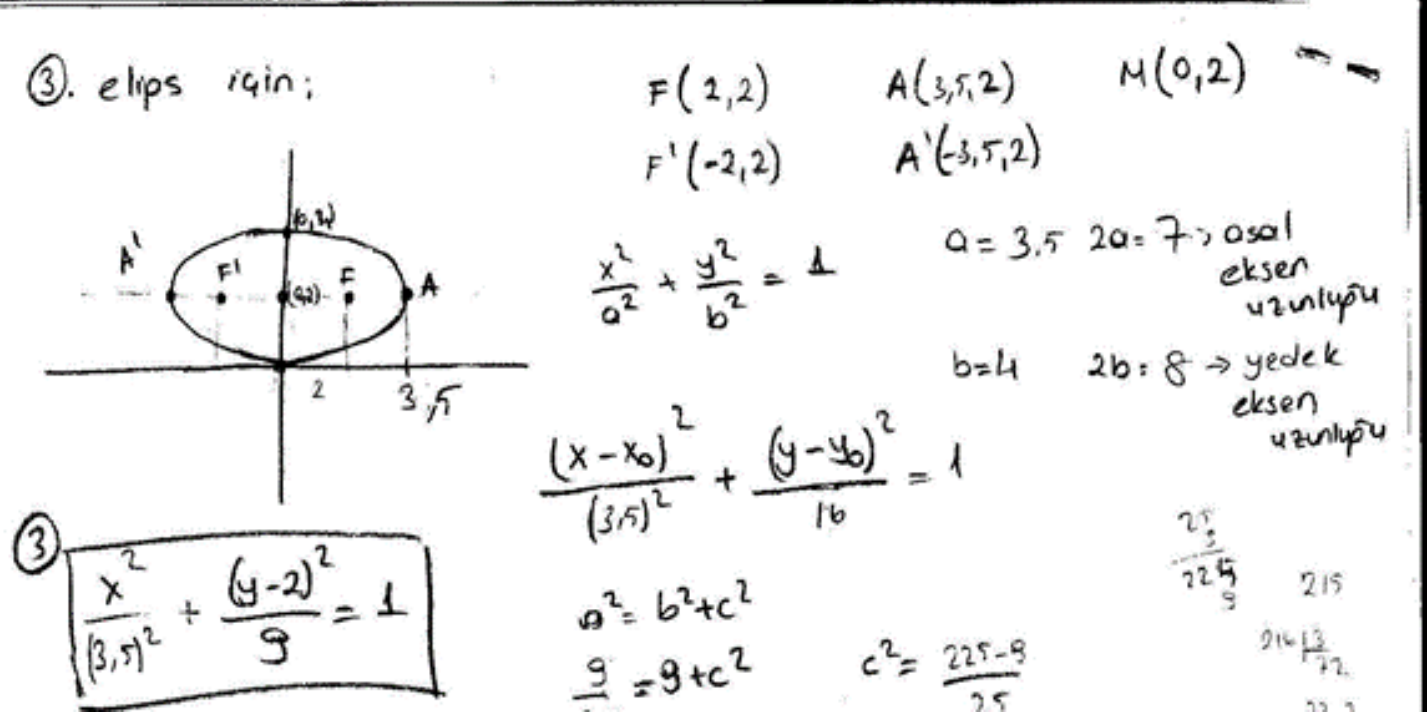

Figure 9. Sample Solution by Teacher Candidate A23

Teacher Candidate A23 found all the three ellipse equations, but when the equations formulated by the participant was checked with Geogebra for verification, the graph below that revealed the difference between the relationship among the ellipses and the given geometric situation was obtained (Figure 10). The teacher candidate clearly did not reach the Evaluating category since s/he did not perform the necessary controls.

The participant broke down the geometric situation into ellipses that formed it, examined the parts one by one and found their equations. However, s/he was evaluated to be in the Analyzing category since $\mathrm{s} /$ he did not make a final check of the relationship between what $\mathrm{s} / \mathrm{he}$ obtained and the whole structure.

\subsection{Results Obtained in the Applying Category}

A total of 18 teacher candidates were evaluated to be in the Analyzing category of higher-order cognitive domain since they found the equations for the ellipses centered at the origin in the coordinate plane that they chose in the given problem situation and found the eccentricities by using the data required for the ellipses.

\section{Sample Solution by Teacher Candidate A28 in the Applying} Category

The solution given by Teacher Candidate A28 (Figure 11) was quoted directly and interpreted below.

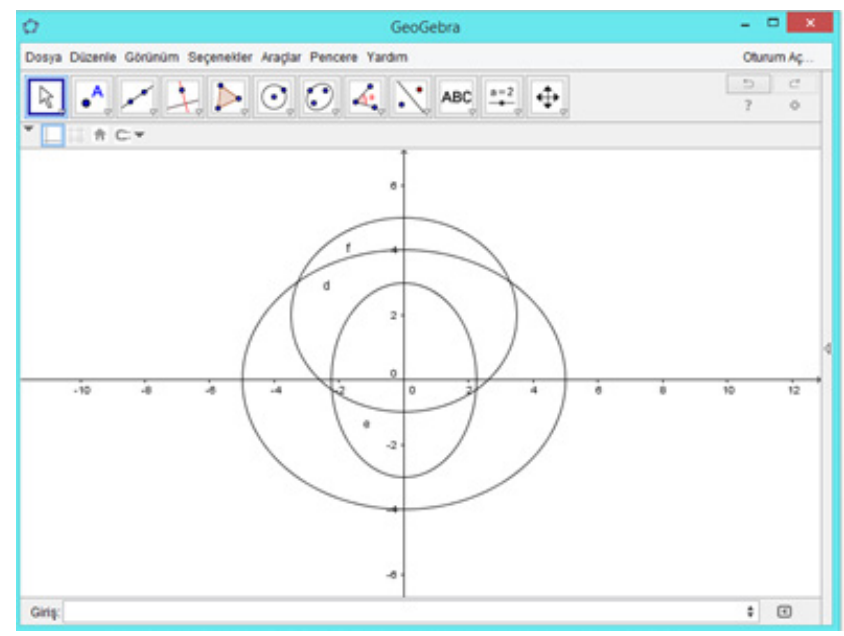

Figure 10. Ggraph of geometric structure formed by Teacher Candidate A23 with the Geogebra software 


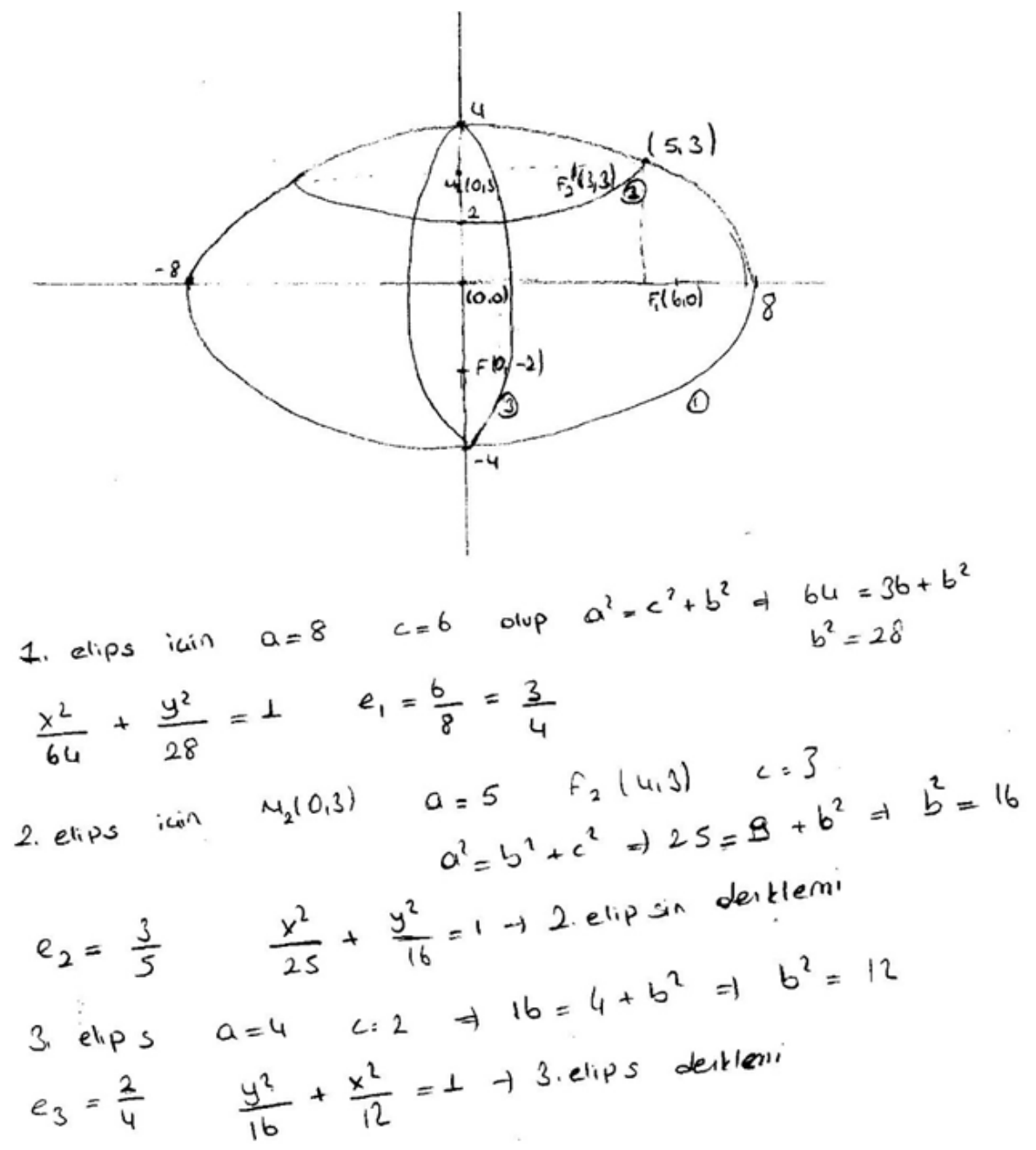

Figure 11. Sample Solution by Teacher Candidate A28

Teacher Candidate A28 plotted the ellipses on a standard coordinate system. The participant found the equation for the larger ellipse but, while formulating the equations for these ellipses, s/he did not consider that one of the two other ellipses needed to be centered at the origin and the other needed to have its axes on y-axis. The participant then calculated the eccentricities of the ellipses by finding the distances between the focuses. Therefore, this teacher candidate was evaluated to be in the Applying category.

All in all, we could suggest that the teacher candidates reached the Applying category at least for each of the situations. In addition, approximately two thirds of the teacher candidates did very well in the implementation stage and reached the higher-order thinking categories of Analyzing, Evaluating and Creating.

\section{Conclusions}

Aiming to explore how mathematics teacher candidates employed their knowledge of the ellipse in the Applying category, a lower order category in the cognitive process dimension, and in the Analyzing, Evaluating and Creating categories, higher-order categories in the cognitive process dimension, this study found that out of 106 teacher candidates 10 participants were in the Creating category, 47 were in the Evaluating category, 31 were in the Analyzing category and 18 were in the Applying category.

For the standard geometric situation given, about half of the teacher candidates plotted the larger ellipse and the vertical ellipse on the coordinate system so that they would be centered at the origin. Taking the relative positions of each of the ellipses into consideration, the participants marked the points where the ellipses intersected the axes and their centers on the coordinate system that they chose. After that, they found the lengths of the major and minor axes for each of the ellipses that were required for writing their equations. Taking the equations, relative positions and (internally tangent) of each of the ellipses into account, they correctly found the equations that yielded compatible values for the lengths of the major and minor axes and represented the relationship for the whole through the parts. The participants also found the values required for eccentricity and calculated eccentricity for each of the ellipses. These teacher candidates were considered to be in the Evaluating category since they were able to interpret the ellipses in 
connection with their flatness.

On the other hand, unlike the teacher candidates in the Evaluating category, some of the participants obtained the three ellipses together as a consistent whole by choosing an original coordinate system or by using letters on a standard coordinate system in the given geometric situation. These participants were evaluated to be in the Creating category of higher-order cognitive domain since they were able to find the eccentricity for each of the ellipses and to interpret the ellipses in connection with their flatness. Having formulated the equations for the three ellipses by assigning letters to the vertices of the ellipses, the teacher candidates finally reached a generalization that represented the situation.

About one-third of the teacher candidates correctly found the equations by using the coordinate system that they determined in the geometric figure formed by the three ellipses and taking the relative positions of only two ellipses into consideration. One of these two ellipses was the larger ellipse centered at the origin and while the other ellipse was either the vertical ellipse whose minor axis was y-axis or the smaller horizontal ellipse that was not centered at the origin. For the equation of the third ellipse at this stage, the teacher candidates either failed to state that the major axis was on the $y$-axis when formulating the ellipse equation or they found the equation for the ellipse that was not centered at the origin as if it had been an ellipse that was centered at the origin. During the implementation stage, the teacher candidates either correctly found the equation for the ellipse that was not centered at the origin or they correctly found the ellipse equations whose major axis was on the y-axis for the questions asking the candidates to find a single ellipse equation. Therefore, these teacher candidates took the relative positions of the two of the three ellipses into account, but they made a mistake when formulating the third equation.

Some of the other teacher candidates divided the given figure into smaller parts, formulated the equations for all the three ellipses separately and connected each part appropriately by considering whether the major axis was on the $\mathrm{x}$-axis, on the $\mathrm{y}$-axis or parallel to the $\mathrm{x}$-axis and whether the ellipses were centered at the origin or not. However, they still failed to reach a judgment by relating each part to the whole. Therefore, they could not reach the Evaluating category and remained in the Analyzing category. On the other hand, those teacher candidates in both categories were evaluated to be in the Analyzing category since they only found the values required for eccentricity, estimated eccentricity and compared them in numbers.

About one-fifth of the teacher candidates plotted the ellipses on the coordinate plane and correctly found only the equation of the ellipse that was centered at the origin. On the other hand, they were able to perform just a single operation in the given situation, but they could not establish a relationship between the other ellipses and the whole. These teacher candidates were evaluated to be in the Applying category. The candidates in this category assigned numerical values to the lengths of the major and minor axes of the ellipses, found the distance between the focuses, which was required for finding the eccentricity, from the equation $a^{2}=b^{2}+c^{2}$, and estimated the eccentricity for each of the ellipses.

The results from our study showed that about two-thirds of the teacher candidates were in Analyzing, Evaluating or Creating categories of higher-order cognitive learning domain in terms of analytically examining ellipses. Those participants in the Analyzing category were able to determine how the ellipses were related to each other and to the overall structure by dividing the holistic geometric model formed by the ellipses in the given figure into smaller parts, but they could not reach a judgment. The participants in the Evaluating category were able to reach a conclusion based on the relationship between the parts and the whole since they were able to infer a judgment through their analyses. Therefore, the participants in the Evaluating category met the criteria for the Analyzing category, and those in the Creating category followed an original approach and obtained the result by making generalizations regarding the relationship for the whole formed by the ellipses. Obviously,

When planning and designing instructional media to equip teacher candidates with higher-order thinking skills, analytical examination of a single ellipse could be followed by sample cases involving analytical examination of multiple ellipses in relation to each other. Promoting teacher candidates' active participation in solving these sample cases and providing them with the opportunity to perform their own reasoning could help them develop higher-order thinking skills.

\section{REFERENCES}

[1] Amer, A. (2006). Reflections on Bloom's Revised Taxonomy. Electronic Journal of Research in Education Psychology, 8, (4): 214-230.

[2] Airasian, P.W., Cruikshank, K.A., Mayer, R.E., Pintrich, P.R., Raths, J., \& Wittrock, M.C. (2001). A taxonomy for learning, teaching, and assessing: A revision of Bloom's Taxonomy of Educational Objectives (Complete edition), Anderson, L.W. (Ed.), Krathwohl, D.R. (Ed.), New York: Longman.

[3] Arı, (2011). Bloom'un gözden geçirilmiş bilişsel alan taksonomisinin Türkiye'de ve uluslararası alanda kabul görme durumu. Kuram ve Uygulamada Eğitim Bilimleri. 11(2), 749-772.

[4] Birgin, O. (2016). Bloom Taksonomisi, E. Bingölbali, S. Arslan, I.O. Zembat (Eds.) Matematik Eğitiminde Teoriler içinde (520-532). Ankara: Pegem Akademi.

[5] Miles, M.B. \& Huberman, A. M., (1994). Qualitative Data Analysis, Sage Publications, London.

[6] Raths, J., \& Wittrock, M.C. (2001). A taxonomy for learning, teaching, and assessing: A revision of Bloom's Taxonomy of Educational Objectives (Complete edition). New York: Longman.

[7] Riddle, D. F. (1996). Analytic Geometry. An International 
Thomson Publishing Company.

[8] Yıldırım, A. ve Şimşek, H. (2005). Sosyal Bilimlerde Nitel Araştırma Yöntemleri. Ankara: Seçkin Yayınları.

[9] Yurdabakan, İ. (2012). Bloon'un Revize edilen Taksonomisinin Eğitimde Ölçme ve Değerlendirmeye Etkileri. Gaziantep Üniversitesi Sosyal Bilimler Dergisi. 11(2),
327-348.

[10] Bloom, B.S. (1956). Taxonomy of educational objectives: The classification of educational goals. New York, NY: Longmans, Green.

[11] Schlechty, P. C. (2011). Engaging students: The next level of working on the work. John Wiley \& Sons. 